\title{
Micro/nano surface texture evaluation by reconstruction from images using approximated BRDF model
}

\author{
Hitendra Kumar ${ }^{1}$, J. Ramkumar ${ }^{1}$ and K. S. Venkatesh ${ }^{2}$ \\ ${ }^{1}$ Department of Mechanical Engineering, Indian Institute of Technology Kanpur, Kanpur -208016, \\ India \\ ${ }^{2}$ Department of Electrical Engineering, Indian Institute of Technology Kanpur, Kanpur -208016, India \\ *Email: jrkumar@iitk.ac.in
}

It is crucial to determine dimension and surface characteristics of a machined part to ensure its optimal functioning. Some applications, for example, biomedical, surgical, precision actuators etc. require the surface to be well engineered. Evaluation of surface roughness of complex free-form parts is one of the major challenge faced in controlling manufacturing processes. Conventional methods use stylus based surface analyzers, which, being contact based, are accurate but slow and cause damage to the surface [1]. Various attempts have been made to estimate surface roughness from non-contact methods by fractal analysis or using statistics from images [2]. Image processing based techniques are more prominent in non-contact methods and use approaches like stereo-vision, structured light or Moiré fringe projection [3, 4]. On the other hand, light reflection models have been used to obtain shape of object from multiple images [5]. Such models are applied on macro-scale analysis but no literature has been reported on their application in micro scale. In this paper, we investigate a method to reconstruct surface of ground specimens from 2D images using a hypothesized function to approximate Bi-directional Reflectance Distribution Function (BRDF). Further, we calculate roughness parameter $\left(\mathrm{Ra}^{*}\right)$ from generated profile and validate against measurements from a stylus based analyzer.

In the proposed model, it is assumed that each pixel captures a very small region such that there is only one surface normal associated with it. As illustrated in Figure 1, a ring-type light source is used to illuminate the surface and capture device is positioned at center of the light source. This arrangement gives angle of incidence and reflection close to $0^{\circ}$ w.r.t. vertical.

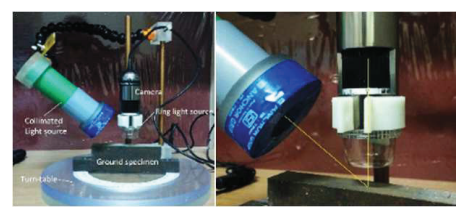

Figure 1: Arrangement of camera, light source and workpiece (left), zoomed image (right)

In order to map observed pixel gray value (255- max., 0 -min.), a function is hypothesized as follows:

$|\tan \theta|=\frac{255-x}{x}$
Where $\theta$ is the angle between surface normal and vertical, 255 is the maximum gray value and $x$ gives the gray value at the pixel. The hypothesized function approximates light intensity distribution of a surface as given by Bi-directional Reflectance Distribution Function (BRDF) [6].

Two parameters, $\mathrm{Ra}$ and $\mathrm{Rq}$, are measured from the generated surface and are validated against measurements from a surface analyzer.
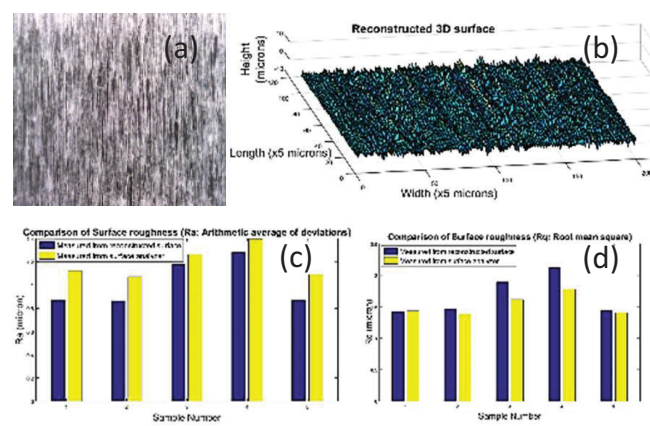

Figure 2: (a) Input image. (b)Reconstructed Surface. (c) Ra parameter comparison. (d) Rq parameter comparison

The paper concludes that the approximate function presents a suitable tool to reconstruct the surface from 2D images and has strong correlation with measured values. This method is more simplified and has the capability to be implemented in online assessment for accurate estimation of surface roughness. However, as the computation is performed on a pixel level, the method computation intensive and also presents some difficulties in handling the slope ambiguities in presence of ambient light.

\section{References}

1. Guerrero JL, Black JT. ASME. J. Eng. Ind. 1972; 94(4):1087-1093.

2. Datta, A., Dutta, S., Pal, S. K., Sen, R., \& Mukhopadhyay,

S. In Advanced Materials Research (Vol. 365, pp. 38-43).

3. Prasad, K. Niranjan, and B. Ramamoorthy. Journal of Materials Processing Technology 112.1 (2001): 43-52.

4. Windecker, Robert, Stefan Franz, and Hans J. Tiziani. Applied optics 38.13 (1999): 2837-2842.

5. Zickler, Todd E., Peter N. Belhumeur, and David J. Kriegman. International Journal of Computer Vision 49.2-3 (2002): 215-227.

6. Prokhorov, Alexander. Applied optics 51.13 (2012): 23222332. 